
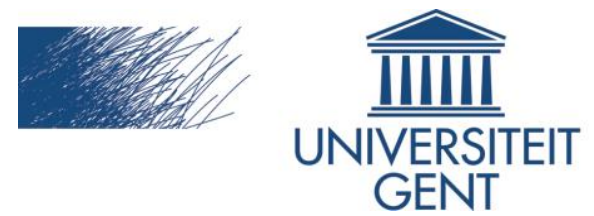

biblio.ugent.be

The UGent Institutional Repository is the electronic archiving and dissemination platform for all UGent research publications. Ghent University has implemented a mandate stipulating that all academic publications of UGent researchers should be deposited and archived in this repository. Except for items where current copyright restrictions apply, these papers are available in Open Access.

This item is the archived peer-reviewed author-version of: Imidazoquinoline-Conjugated Degradable Coacervate Conjugate for Local Cancer Immunotherapy

Authors: Li H., Van Herck S., Liu Y., Hao Y., Ding X., Nuhn L., Zhong Z., Combes F., Sanders N., Lienenklaus S., Koker S.D., David S.A., Wang Y., De Geest B., Zhang Z.

In: ACS Biomaterials Science \& Engineering 6(9): 4993-5000

To refer to or to cite this work, please use the citation to the published version:

Li H., Van Herck S., Liu Y., Hao Y., Ding X., Nuhn L., Zhong Z., Combes F., Sanders N., Lienenklaus S., Koker S.D., David S.A., Wang Y., De Geest B., Zhang Z. (2020)

Imidazoquinoline-Conjugated Degradable Coacervate Conjugate for Local Cancer Immunotherapy

ACS Biomaterials Science \& Engineering 6(9): 4993-5000

DOI: 10.1021/acsbiomaterials.0c00485 


\section{An Imidazoquinoline-Conjugated Degradable Coacervate Conjugate for Local Cancer Immunotherapy}

Hui Li, ${ }^{\dagger}$ Simon Van Herck,, Yongjun Liu, ${ }^{\dagger}$ Yanyun Hao, ${ }^{\dagger}$ Xiaochu Ding, ${ }^{\S}$ Lutz Nuhn, " Zifu Zhong, ${ }^{\perp}$ Francis Combes, ${ }^{\perp}$ Niek N. Sanders, ${ }^{\perp}$ Stefan Lienenklaus,,${ }^{\prime}$ Stefaan D. Koker,, Sunil A. David, ${ }^{\forall}$ Yadong Wang, ${ }^{\S}$ Bruno G. De Geest, ${ }^{*}{ }^{\star}$ Zhiyue Zhang, ${ }^{*}+$

† Department of Pharmaceutics, Key Laboratory of Chemical Biology (Ministry of Education), School of Pharmaceutical Sciences, Cheeloo College of Medicine, Shandong University, 44 Wenhuaxi Road, Jinan, Shandong Province 250012, China.

‡ Department of Pharmaceutics, Ghent University, Ghent, Belgium.

$\S$ Nancy E and Peter C. Meinig School of Biomedical Engineering, Cornell University, New York 14853, United States.

|| Max Planck Institute for Polymer Research, Ackermannweg 10, 55128 Mainz, Germany.

$\perp$ Laboratory of Gene Therapy, Department of Nutrition, Genetics and Ethology, Faculty of Veterinary Medicine, Ghent University, Belgium.

\# Institute for Laboratory Animal Science, Hannover Medical School, Hannover, Germany.

$\forall$ ViroVax LLC 5950 Research Parkway, Lawrence, KS 66047, United States. 


\section{ABSTRACT}

Strategies that can reduce harmful side effects of potent immunomodulatory drugs are in high demand to facilitate clinical translation of the newest generation of immunotherapy. Indeed, uncontrolled triggering of the immune system can lead to life-threatening cascade reactions such as e.g. cytokine storm. In particular, drug formulations that combine simplicity and degradability are of formidable relevance. Imidazoquinolines are an excellent example of such small molecule immunomodulatory drugs that exhibit in unformulated form a highly undesirable pharmacokinetic profile. Imidazoquinolines are potent inducers of type I interferons that are of great interest in the context of anti-cancer and anti-viral therapy through triggering of Toll like receptors 7 and 8 . In this paper we aimed to alter the pharmacokinetic profile of imidazoquinolines using a simple, yet efficient, strategy that holds high potential for clinical translation. Hereto, we conjugated an imidazoquinoline to the backbone of poly(aspartate) and further formulated this into a degradable coacervate through complex coacervation with a non-toxic degradable polycation. The intrinsic TLR activity of the imidazoquinoline was well preserved and our formulation strategy offered spatial control over its biological activity in vivo

\section{INTRODUCTION}

Small molecule drugs that can provoke innate immune activation through triggering of specific receptors expressed by innate immune cell subsets are of great relevance for cancer immunotherapy and vaccine development. ${ }^{1}$ However, the major obstacles for clinical translation of many of these molecules are their poor physicochemical and pharmacokinetic properties in vivo affording dose-limiting toxicities. Hence, strategies that can reduce unwanted side effects of potent immunomodulatory drugs are in high demand to facilitate clinical translation of the newest generation of immunotherapeutics. Indeed, uncontrolled triggering of the immune system can lead to life-threatening cascade reactions such as cytokine storm. ${ }^{2}$ Additionally, non-degradable by-products from specifically designed drug delivery systems can be prone to long-term accumulation. Therefore, drug formulations that combine simplicity and degradability are of great relevance. ${ }^{3,4}$ 
Toll like receptors (TLRs) are a class of natural immune pattern recognition receptors, which can mediate both natural and adaptive immunity in humans and rodents. ${ }^{5-7}$ Most TLRs can activate the host defense system, produce IL-1 $\beta$, IL-6, TNF- $\alpha$, and chemotactic cytokines, thereby regulating the Th1/Th2 balance. ${ }^{8}$ In recent years, TLR agonists have attracted much attention as adjuvants or immunomodulators for tumor immunotherapy. ${ }^{9}$, ${ }^{10}$ Single stranded viral RNA is the natural ligand of TLR7/8 and is a potent trigger of antiviral and anti-tumoral responses. ${ }^{11}$ Interestingly, a wide range of potent small molecule agonists of TLR7/8 have been discovered but are an exquisite example of immunomodulatory drugs that in unformulated state rapidly distribute throughout the whole body and cause systemic inflammation. TLR7/8 are localized on the endosomal membrane of innate immune cells which are therapeutic targets to promote antigen presentation and mount tumor-specific adaptive immunity. ${ }^{12}$ Previously, we and others have demonstrated that covalent conjugation of imidazoquinolines to macromolecular or colloidal carriers strongly abrogates systemic dissemination but rather confers the type I interferon response to the site of administration and to draining lymphoid tissue..$^{13-17}$ The latter is of particular interest in the context of using these systems for localized cancer immunotherapy or as a vaccine adjuvant. Immune cells located in specific areas of lymphoid tissue not only respond to antigens from distal infection, but also receive antigen from antigen presenting cells that are recruited from nearby tumor or a site of infection.

In this paper we aimed to alter the unwanted pharmacokinetic profile of imidazoquinolines using a simple, yet efficient, strategy that holds high potential for clinical translation. Hereto we conjugated an imidazoquinoline to the backbone of the biodegradable polypeptide poly(L-aspartic acid) at its primary amine position to offer spatial controlling, and further formulated this conjugation into a degradable coacervate through complex coacervation with a non-toxic degradable polycation to circumvent systemic immune-stimulation. Importantly, no multi-step reaction or toxic solvents/reagents were required during formulation which makes it safe and biocompatible. The intrinsic TLR activity of the imidazoquinoline could be well preserved and its biological activity in vivo spatially confined to the local site of administration. 


\section{RESULTS AND DISCUSSION}

\section{Synthesis and characterization}

Polyaspartic acid (PASP) is a biodegradable polypeptide, that was synthesized from polysuccinimide (PSI) as a precursor polymer (Scheme 1). In a first step, polysuccinimide (PSI) was synthesized by acid-catalysed polycondensation of aspartic acid, ${ }^{18,}{ }^{19}$ resulting in a polymer with a number average molar mass of $6.2 \mathrm{KDa}$ and a dispersity $(\Theta)$ of 1.49 (according to SEC in DMAc), with only $3 \%$ of branched polymer formed (according to ${ }^{1} \mathrm{H}$ NMR) (Figure S1-3). In a subsequent step, the resulting PSI was treated with aqueous sodium hydroxide, which induces opening of the succinimide ring. After purification via dialysis against demi water, successful synthesis of PASP was evidenced by ${ }^{1} \mathrm{H}-\mathrm{NMR}$ spectroscopy, containing $75 \%$ of beta form and $25 \%$ of alpha form along the polymer chain (according to ${ }^{1} \mathrm{H}-\mathrm{NMR}$ ) (Figure S4, S5). Contrary to solid phase synthesis or ringopening polymerization of $\mathrm{N}$-carboxyanhydride monomers, this route excels in simplicity and does neither require the use of multiple reaction and deprotection cycles (applied in solid phase synthesis) nor hazardous chemicals or carefully monitored reaction conditions (as for NCA polymerization). The resulting polypeptide is equipped with a relatively moderate dispersity, thus, making it also highly attractive for large scale industrial production and applications.

Next, the TLR7/8 agonist IMDQ was covalently conjugated to the polymer through amide bond formation between the primary amine of the IMDQ and carboxylic acid moieties on the PASP backbone using DMTMM to facilitate amide bond formation. ${ }^{20}$ When needed for further fluorescence-based assays, fluorescently labelled PASP-IMDQ was prepared by conjugating an amine-bearing fluorescent dye to the PASP backbone in a similar fashion as for IMDQ. Finally, after purification by dialysis, PASP-IMDQ could be isolated in dry form by lyophilization. The absence of free IMDQ was confirmed by HPLC analysis (Figure S6) and the amount of IMDQ in PASP-IMDQ was calculated based on UV-Vis spectroscopy to be $15 \%(\mathrm{w} / \mathrm{w})$ (Figure S7). 
<smiles>CC(C)[C@H](C)C1CC(=O)N(C(C)(C)C)C1=O</smiles>

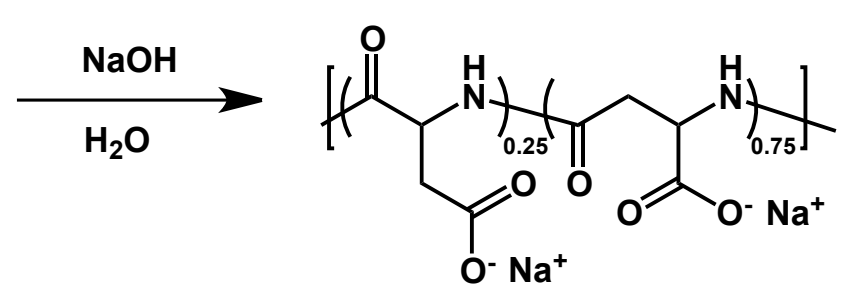
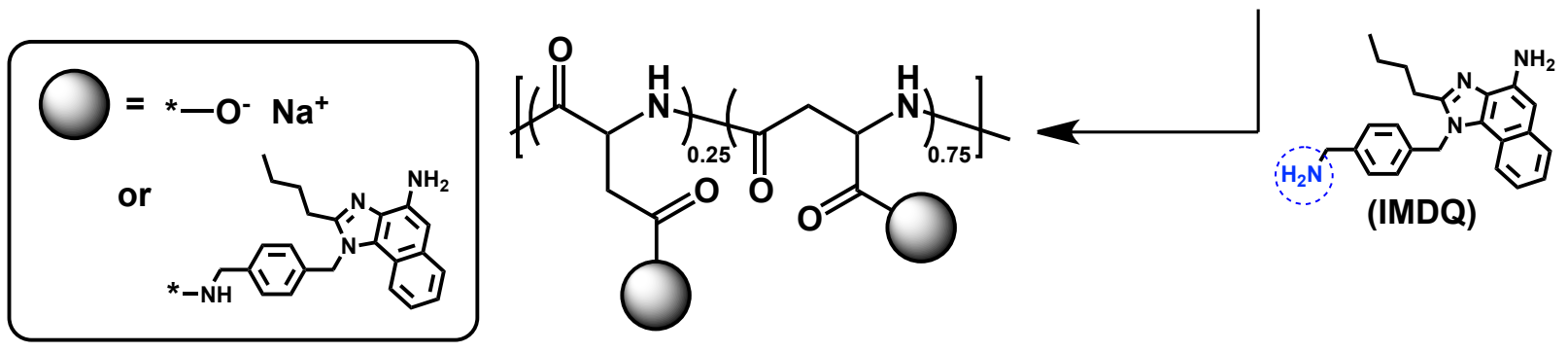

Scheme 1. Synthesis of poly(aspartate) (PASP) and conjugation of the TLR7/8 agonist IMDQ. (A) polysuccinimide (PSI) was synthesized by acid-catalysed polycondensation of L-aspartic acid. (B) PSI was treated with $\mathrm{NaOH}$, which yields opening of the succinimide rings. (C) the TLR7/8 agonist IMDQ was covalently conjugated to the polymer through amide bond formation between primary amino group of the IMDQ and carboxylic acid group of the PASP facilitated by DMTMM.

\section{In vitro cell uptake and innate immune activation}

First, the cytotoxicity of the polymer was evaluated by MTT assay, revealing PASPIMDQ to be non-toxic up to a concentration of $13 \mu \mathrm{M}$ (i.e. $>100 \mu \mathrm{g} / \mathrm{mL}$ ) (Figure $1 \mathrm{~A}$ ). Next, we examined the in vitro uptake of the polymer by mouse RAW macrophages as model innate immune cells. Because TLR7/8 is localized on the endosomal membrane, PASPIMDQ must be endocytosed by the cells in order for IMDQ to reach its target receptor. ${ }^{12}$ RAW macrophages were pulsed with rhoPASP-IMDQ (i.e. bearing a rhodamine label) followed by flow cytometry (FACS) analysis and confocal microscopy imaging. FACS analysis revealed that rhoPASP-IMDQ associated to cells in a dose-dependent manner (Figure 1B). Confocal microscopy confirmed that the polymer was indeed internalized by the macrophages, the punctuated fluorescence pattern hinting at accumulation in endolysosomal vesicles (Figure 1C), which is in line with our earlier findings with regard to subcellular localization of macromolecular IMDQ conjugates. ${ }^{14}$

Next, we set out to determine whether PASP-IMDQ can trigger TLR activation. Hereto we used the RAW Blue pan TLR-reporter cell assay. The latter allows for quantitative 
determination of TLR agonistic activity by simple spectrophotometric detection of SEAP enzymatic activity. As shown in Figure 1D, control PASP (i.e. without IMDQ) did not induce activation, suggesting that the polymer on its own is poorly immunogenic, at least under the experimental conditions. By contrast, PASP-IMDQ strongly promoted TLRactivation at an almost equal potency as IMDQ in soluble form. These findings are interesting as in our previous work on macromolecular IMDQ-conjugates, typically a 2-3 log loss in activity was observed when IMDQ was conjugated to a carrier, relative to IMDQ in soluble form, likely due to sterical hindrance caused by the macromolecular carrier. We hypothesize that the polypeptide backbone could be prone to degradation by endosomal peptidases upon cellular uptake, which would result in smaller fragments that pose less sterical hindrance to IMDQ for reaching the target binding pocket.
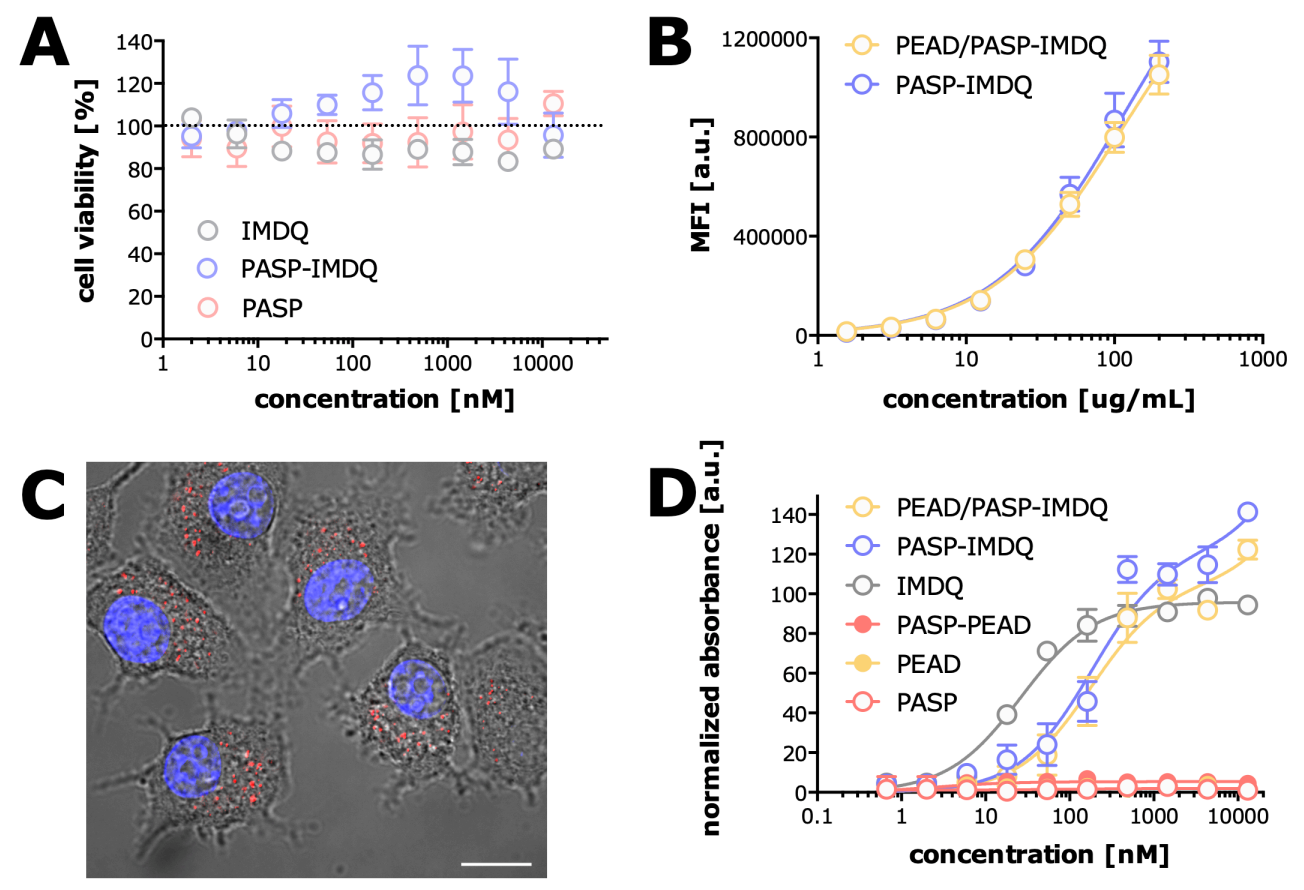

Figure 1. In vitro cellular uptake and innate immune activation of PASP-IMDQ. (A) Cytotoxicity (assessed by MTT assay) on RAW Blue macrophage pulsed with native soluble IMDQ, PASP, PEAD and PASP-IMDQ, as well as coacervated PEAD/PASP and PEAD/PASP-IMDQ $(n=6)$. (B) Flow cytometry analysis of RAW Blue macrophage pulsed with rhoPASP-IMDQ (i.e. bearing a rhodamine label) and coacervated PEAD/ rhoPASP-IMDQ ( $n=3)$. (C) Corresponding confocal microscopy image of RAW Blue macrophage pulsed with rhoPASP-IMDQ. Scale bar represents 15 micron. (D) RAW Blue pan TLR-reporter cell assay (by spectrophotometric detection of SEAP enzymatic activity) measuring TLR agonistic activity of native soluble IMDQ, PASP, PEAD and PASP-IMDQ, as well as coacervated PEAD/PASP and PEAD/PASP-IMDQ $(n=6)$. 


\section{Complex coacervation}

To allow for a more prolonged stimulation in the tumor microenvironment we reasoned that complex (electrostatic) coacervation of PASP-IMDQ with a degradable cationic polymer could be a viable option. Poly(ethylene argininylaspartate diglyceride) (PEAD), is a non-toxic biodegradable polycation with high biocompatibility and which has previously been shown to exhibit high affinity towards biological polyanions such as DNA, heparin and hyaluronic acid, forming complex coacervate droplets. ${ }^{21,} 22$

At first we attempted to derive the optimal conditions for complex coacervation of PASP$I M D Q$ and PEAD. Testing several concentration and ratios of both compounds resulted in selecting a 5:1 (w/w) ratio of PEAD to PASP-IMDQ at a PEAD concentration of 25 $\mathrm{mg} / \mathrm{mL}$ and a PASP-IMDQ concentration of $5 \mathrm{mg} / \mathrm{mL}$ as to be optimal in terms of PASPIMDQ encapsulation (i.e. 90\%) while limiting the excess of free PEAD (Figure S8). Figure $2 A$ shows a confocal microscopy image of the PEAD/ rhoPASP-IMDQ system which clearly shows the presence of coacervate droplets with a size between 1-20 micron. To measure the release of PASP from the coacervate we incubate the PEAD/rhoPASPIMDQ in phosphate buffered saline (PBS) at $\mathrm{pH} 7.4$ and $37^{\circ} \mathrm{C}$, followed by sample collection, centrifugation and determination of the amount of rhoPASP in the supernatant. As shown in Figure 2B, a sustained release for up to $12 \mathrm{~h}$ in vitro is achieved by coacervation of PASP with PEAD. However, it is important to note that in this experiment the coacervate was suspended in aqueous medium, whereas upon injection in tissue the excess of water will diffuse and the coacervate will form a tightly packed gel which we hypothesize will presumably exhibit significantly slower PASP-IMDQ release kinetics. Flow cytometry analysis showed no difercence in cellular asosciatiom between rhoPASPIMDQ and PEAD/rhoPASP-IMDQ (Figure 1B), which we attribute to rhoPASP-IMDQ being readily released from the coacervate droplets under the diluted conditions used for in vitro experiments. Similar findings were observed when assessing the TLR agonistic acitivy of PEAD/rhoPASP-IMDQ (Figure 1D), proving that coacervate-formulated IMDQ remains a potent immunomodulator with an EC50 in the nanomolar range. 

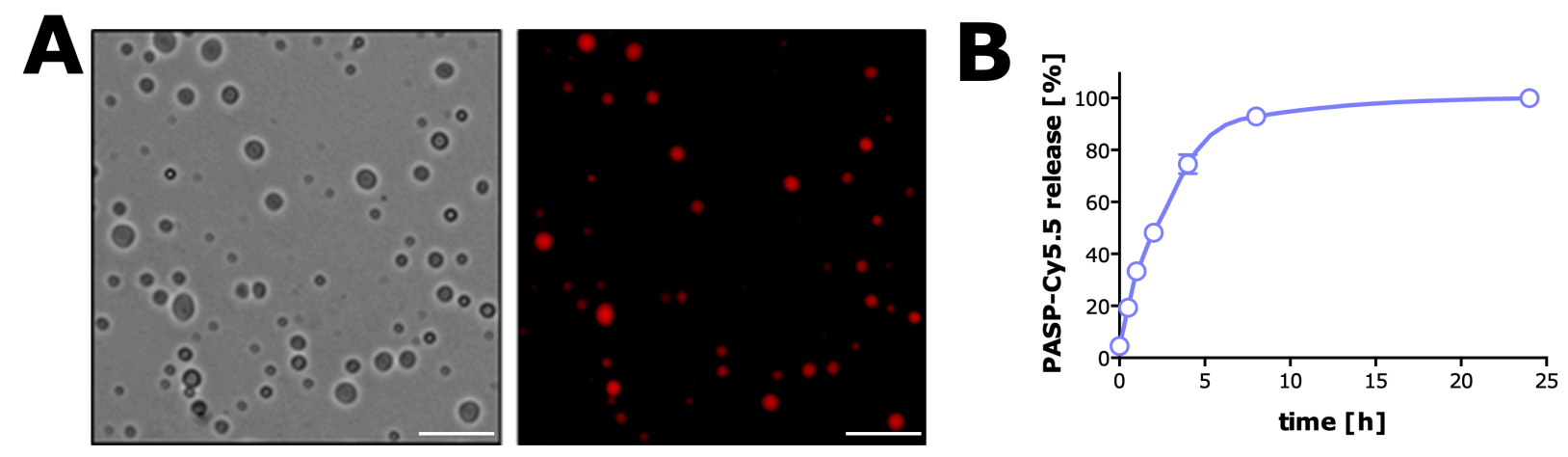

Figure 2. Characteristics of $P E A D / P A S P-I M D Q$ system. (A) A confocal microscopy image of the PEAD/ rhoPASP-IMDQ coacervate. Scale bar represents 20 micron. (B) Release of Cy5.5PASP from the coacervate PEAD/ Cy5.5PASP in phosphate buffered saline at $\mathrm{pH} 7.4$ and $37^{\circ} \mathrm{C}$.

\section{In vivo innate immune activation}

To assess to what extent our formulation strategy influences the spatial distribution of innate immune activation by PASP-IMDQ we made use of an IFN- $\beta$ luciferase (IFN $\beta+/ \Delta \beta$ luc) reporter mouse model. In this model, a firefly luciferase reporter gene is linked to the type I interferon, IFN- $\beta .{ }^{23}$ Mice were injected intradermally in the flank with an equivalent dose of $10 \mu \mathrm{g}$ of IMDQ in soluble and in PEAD/PASP-IMDQ form, followed by noninvasive full body luminescence imaging. As depicted in Figure $3 \mathrm{~A}$, a strong systemic IFN- $\beta$ response was induced by soluble IMDQ with a profound expression of IFN- $\beta$ in a variety of major organs, which is likely due to rapid diffusion of IMDQ from injected site into the circulation. By contrast, PEAD/PASP-IMDQ induced a strongly localized immunomodulatory response. We then calculated the ratio of local to the total luminescence, which pointed out the ability of PEAD/PASP-IMDQ to supress systemic inflammatory responses and rather restrict its biological activation at the site of administration (Figure 3B). 


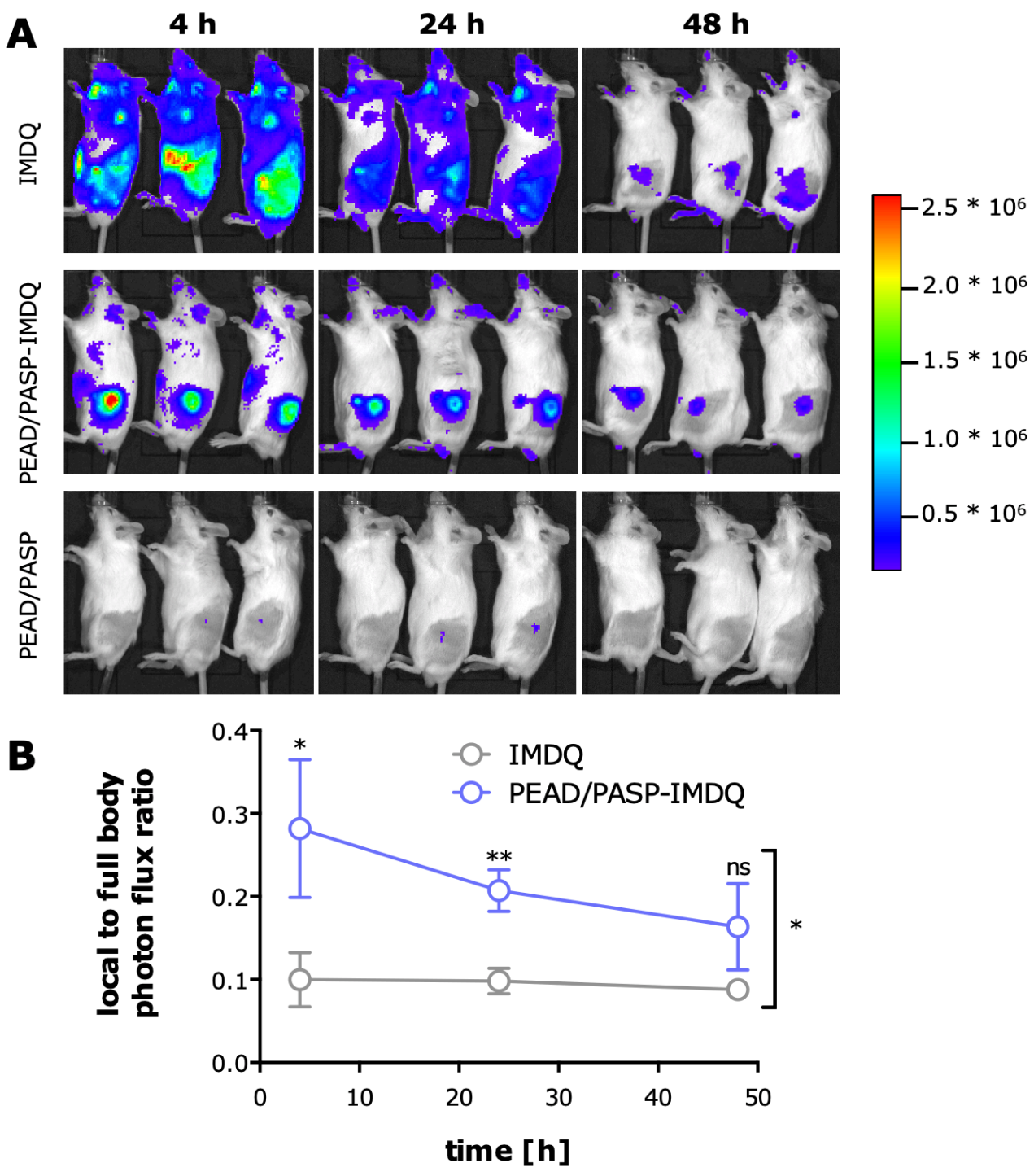

Figure 3. (A) In vivo luminescence images of IFNß-luciferase reporter mice that received intradermal injection in the flank with $10 \mu \mathrm{g}$ of IMDQ and PEAD/PASP-IMDQ (empty PEAD/PASP was included as control). (B) Quantification of the luminescence data. ( $n=3$; Student t-test on AUC: *: $p<0.05$ )

\section{Anti-tumoral activity}

Recent preclinical and clinical studies indicate that intratumoral administration of TLR agonists stimulates antigen-presenting cells and helps reverse the immunosuppressive microenvironment that protects large established tumors from immune elimination. The activation of antigen-presenting cells (such as dendritic cells (DCs)) results in the production of proinflammatory cytokines, and supports antigen presentation to CD8 T 
cells in lymphoid tissue thereby promoting anti-tumor immunity. ${ }^{24}$ As a monotherapy, the administration of TLR7/8 agonists conjugates or loaded nanoparticles leads to controlled tumor growth. ${ }^{9}, 23$ Encouraged by these findings, we engaged into assessing the therapeutic potential of PASP-IMDQ in a cancer immunotherapy context. For this purpose, we made use of the murine CT26 colon carcinoma model, which is characterized by a highly immunogenic tumor microenvironemt, expressing numerous neo-antigens, and which has become a popular platform model for evaluating therapeutic stretgaies involving innate and adaptive immune activation.

To test the anti-tumor efficacy of PEAD/ PASP-IMDQ, CT26 mouse colon cancer tumors were grown in BALB/C mice and when a volume of around $100 \mathrm{~mm}^{3}$ was reached, mice were treated by intratumoral injection. Figure 4 depicts the effect of treatment on tumor growth as a function of time. When given repeatedly in native form from or in PASPIMDQ conjugated form, IMDQ only induced a minor decrease in tumor growth. However, mice treated with PEAD-PASP-IMDQ coacervate showed a significant decrease in tumor growth. Eralier, we have demonstrated in a B16 mouse melanoma model that innate immune activation in the tunor microenvironment by intratuoral administration of IMDQ induces activation of DCs in sentinel lymph nodes and presentation of tumor antigen to $T$ cells, thereby inducing robust proliferation of antigen-specific CD9 T cells. In the context of the present study, our data strongly point at linking the ability of PEAD/ PASP-IMDQ to induce prolongued innate immune stimulation in the tumor microenvironment (cfr. Figure 3) to the induction of anti-tumor adaptive immunity. In parallel, we monitored the body weight of the animals (Figure S9) which was found to remain stable over the duration of the experiment, thereby suggesting that the treatment was fairly well tolerated. 


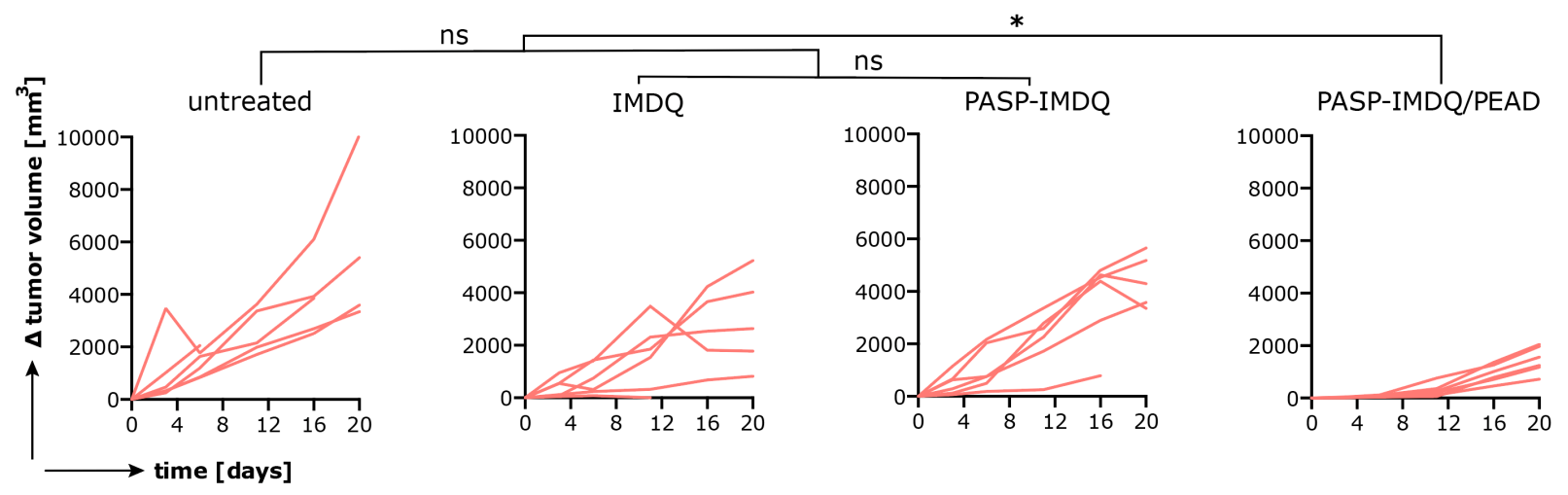

Figure 4. Therapeutic effect on tumour growth as a function of time. CT-26 mouse colon cancer tumours were grown in BALB/C mice and when a volume of $100 \mathrm{~mm}^{3}$ was reached, mice were treated by intratumoral injection of 50 $\mu \mathrm{L}$ of IMDQ, PASP-IMDQ and PEAD/ PASP-IMDQ. $(\mathrm{n}=6$ : Student $\mathrm{t}$-test on AUC: $*: p<0.05)$

\section{CONCLUSIONS}

In conclusion, we have shown in this work that conjugation of IMDQ, a highly potent immune-modulatory drug, to poly(aspartate) (i.e. PASP-IMDQ) is a highly attractive strategy to on the one hand preserve the intrinsic TLR agonistic activity of IMDQ, while strongly restricting the biological activity in vivo to the local site of administration. When formulating PASP-IMDQ into a coacervate through complex coacervation with PEAD, a non-toxic degradable polycation, a potent anti-tumoral effect is observed, attributed to prolongued innate immune stimulation in the tumor microenvironemt. Our findings highlight PEAD/ PASP-IMDQ as a safe type of immunotherapy with - owing to its chemical simplicity and easy preparation - high translational potential.

\section{MATERIALS AND METHODS}

Materials. Unless otherwise stated, all chemicals were purchased from Sigma Aldrich. Tetramethylrhodamine(TMR) cadaverine was obtained from Life Technologies. The TLR7/8 agonist IMDQ (1-(4-(aminomethyl)benzyl)-2-butyl-1H-imidazo[4,5-c]quinolin-4amine) was synthesized as earlier reported. ${ }^{25}$ Dialysis was performed using Spectra/Por 3 membranes obtained from Spectrum Labs. The RAW Blue cell line and Quanti blue 
stain was obtained from Invivogen. Cell culture medium and supplements, Hoechst, and Zeocin were purchased from Invitrogen.

Cells were cultured in RPMI Medium 1640 supplemented with $10 \%$ heat-inactivated fetal bovine serum, $1 \%$ penicillin/streptomycin, $2 \times 10^{-3} \mathrm{M} \mathrm{L}$-glutamine, $1 \times 10^{-3} \mathrm{M}$ sodium pyruvate and $0.01 \%$ Zeocin and incubated at $37{ }^{\circ} \mathrm{C}$ with $5 \% \mathrm{CO}_{2}$ saturation. Mouse experiments were conducted following the European guidelines for animal experiments and approved by the local ethical committee for animal experiments of Ghent University (EC2018/92). Female BALB/c mice (weight: 18-22 g), in the range of 6-8weeks, were supplied by the Medical Animal Test Center of Shandong University (Jinan, China). The animals were fed with a standard diet and allowed water ad libitum. All experiments were carried out in compliance with the Animal Management Rules of the Ministry of Health of the People's Republic of China (document number 55, 2001) and the Animal Experiment Ethics Review of Shandong University.

Synthesis of poly-L-aspartic acid. To prepare the polysuccinimide precursor polymer, first L-aspartic acid $(25 \mathrm{~g}, 0.188 \mathrm{~mol})$ and phosphoric acid $(1.862 \mathrm{~g}, 0.019 \mathrm{~mol})$ was dissolved in $56 \mathrm{~mL}$ mesitylene and $24 \mathrm{~mL}$ DMF in a round-bottom flask and refluxed for $24 \mathrm{~h}$ under nitrogen atmosphere. Next, the solvent was removed by evaporating at reduced pressure and the residue was dissolved in DMF followed by precipitation in water. The precipitate was washed with water several times until neutral. The final product was dried under vacuum at $80^{\circ} \mathrm{C}$ for $24 \mathrm{~h}$.

To hydrolyze the succinimide motifs, $\mathrm{PSI}$ was added to an aqueous solution of $\mathrm{NaOH}$ $(0.2 \mathrm{M})$ while stirring at room temperature for $24 \mathrm{~h}$. The $\mathrm{pH}$ of the resulting solution was neutralized by addition of an aqueous $\mathrm{HCl}$ solution $(0.1 \mathrm{M})$, followed by dialyzed against demi water (MWCO 3.5KDa). The sodium salt of poly(aspartic acid) was isolated in dry form by freeze-drying.

${ }^{1} \mathrm{H}-\mathrm{NMR}$ spectra were recorded on a Bruker $300 \mathrm{MHz}$ or $400 \mathrm{MHz}$ FT NMR spectrometer. Chemical shifts $(\delta)$ are provided in ppm relative to TMS. 
Molecular weight and dispersity of the precursor polymer PSI was measured by size exclusion chromatography (SEC) using dimethylacetamide(DMAc) containing $50 \mathrm{mM}$ $\mathrm{LiBr}$ as eluent. The SEC system consisted of a 20A ISO-pump, a 20A refractive index detector (RID) and an AS 1555 auto sampler. Measurements were recorded at $50^{\circ} \mathrm{C}$ with a flow rate of $0.7 \mathrm{~mL} / \mathrm{min}$. Calibration of the $2 \mathrm{PL} 5 \mu \mathrm{m}$ Mixed-D columns was done with poly(methyl methacrylate) (PMMA) standards obtained from PSS (Mainz, Germany).

Conjugation of IMDQ to poly-L-aspartic acid (PASP-IMDQ). PASP-IMDQ was synthesized through amide bond formation between carboxylic acid motifs on the PASP backbone and the aliphatic amine of IMDQ. Briefly, to a $10 \mathrm{mg} / \mathrm{mL}$ solution of PASP in a phosphate saline buffer (PBS) $(10 \mathrm{~mL}), 1.5$ molar excess of DMTMM in relation to IMDQ was added followed by $10 \mathrm{mg}$ of IMDQ dissolved in DMSO $(10 \mathrm{mg} / \mathrm{mL})$ under continuous stirring. After reaction for three days at room temperature, the solution was transferred into a dialysis membrane (MWCO: $3.5 \mathrm{KDa}$ ) and dialyzed against $0.1 \mathrm{M} \mathrm{NaCl}$ for three days. Subsequent dialysis against demi water for $8 \mathrm{~h}$ and lyophilization afforded PASPIMDQ as a dry powder.

In case of fluorescent labelling, $50 \mu \mathrm{L}$ of amine-containing fluorescent dye $(10 \mathrm{mg} / \mathrm{mL}$ in DMSO) was added after addition of the IMDQ in the above-described synthesis protocol.

To confirm successful conjugation of IMDQ to the PASP backbone and to confirm removal of freely soluble IMDQ by the dialysis step, high-performance liquid chromatography (HPLC) analysis was performed using a Merck, Hitachi LaChrom HPLC system equipped with an isocratic solvent pump (L-7100) set at a flow rate of $1 \mathrm{~mL} / \mathrm{min}$, an autosampler (L-7200) with a loop of 100microL, a guard column (RP 18e) followed by a reversed phase C18 column (LiChroCart $® 250-4$, LiChrospher ${ }^{\circledR} 100 \mathrm{RP}(5 \mu \mathrm{m})$ ) and a UV-detector (L-7400) set at a wavelength of $250 \mathrm{~nm}$. The mobile phase consisted of $65 \%$ water-35\%acetonitrile (supplemented with $0.1 \%(\mathrm{~V} / \mathrm{V})$ trifluoroacetic acid) and the injection volume was set at $10 \mu \mathrm{L}$.

The amount of TLR7/8 agonist IMDQ attached to the polymer was determined by UVVis spectroscopy. Briefly, samples were dissolved in PBS at known concentrations and added to quartz cuvettes with a path length of $1 \mathrm{~cm}$. UV-Vis spectra were recorded on a 
Shimadzu UV-1650PC spectrophotometer over a spectrum from 250-450nm. The amount of IMDQ was calculated based on the obtained calibration curve of IMDQ as a function of concentration.

Coacervate formation. PEAD was synthesized as previously described by the Wang's lab. ${ }^{26}$ Briefly, PEAD was synthesized by polycondensation of aspartic acid and ethylene glycol diglycidyl ether (EGDE), followed by the conjugation of arginine which provided positive charges to the polymer.

Under physiological conditions, PEAD has two cationic groups (amine and guanidine) per repeating unit, and should interact strongly with negatively charged macromolecules through coulombic attraction. Upon addition of PEAD to PASP solution, the solution became turbid and the highest turbidity was reached with a PEAD to PASP mass ratio of $5: 1$, indicating that the PEAD/PASP complex is likely neutral at this ratio. PEAD was dissolved at $25 \mathrm{mg} / \mathrm{mL}$ in demi water and mixed with PASP at a concentration of $5 \mathrm{mg} / \mathrm{mL}$.

\section{In vitro experiments.}

Flow cytometry. RAW mouse macrophages were seeded in a 24-well plate at a density of 250,000 cells $\mathrm{mL}^{-1}$ one day before the cells were pulsed with rhoPASP-IMDQ and $P E A D /$ rhoPASP-IMDQ coacervate at different concentrations. After $24 \mathrm{~h}$ of incubation, the macrophages were dissociated using cell dissociation buffer followed by centrifugation for $5 \mathrm{~min}$ at $200 \mathrm{G}$. Flow cytometry analysis was performed on a BD Accuri. Data were processed using the FlowJo software package.

Confocal microscopy. RAW mouse macrophages were seeded in a glass bottom Willco dish at a density of 250,000 cell mL $\mathrm{mL}^{-1}$ and incubated overnight before the cells were pulsed with rhoPASP-IMDQ. After $24 \mathrm{~h}$ incubation, cells were fixated in a $4 \%$ paraformaldehyde solution for $30 \mathrm{~min}$ at $37^{\circ} \mathrm{C}$ and subsequently washed and simultaneously stained by Hoechst for $1 \mathrm{~h}$ at room temperature. Finally, the samples were washed with PBS and imaged by a confocal microscope (Leica DMI6000 B inverted 241 microscope) equipped with an oil immersion objective (Zeiss, 63×, NA 1.40) and attached 
to an Andor DSD2 confocal scanner. Images were processed using the ImageJ software package.

In vitro RAW Blue assay. RAW Blue macrophages were seeded in a 96-well round bottom plate with $180 \mu \mathrm{L}$ of cells suspension in each well at a density of 50,000 cells $\mathrm{mL}^{-}$ 1 and immediately pulsed with $20 \mu \mathrm{L}$ of test compounds at desired concentrations in sixfold. As a negative control, PBS was added. After $24 \mathrm{~h}$ incubation, $50 \mu \mathrm{L}$ of the supernatant was transferred into a 96-well flat bottom plate and incubated with $150 \mu \mathrm{L}$ of Quanti blue solution. After 0.5 to $2 \mathrm{~h}$ incubation at $37^{\circ} \mathrm{C}$, the color change absorbance was measured with a plate reader at $620-655 \mathrm{~nm}$.

MTT assay. Cell viability was assessed by the 3-(4,5-dimethylthiazol-2-yl)-2,5diphenyltetrazolium bromide (MTT) assay. RAW mouse macrophages were seeded in a 96-well plate with $100 \mu \mathrm{L}$ of cells suspension in each well at a density of 50,000 cells $\mathrm{mL}^{-}$ 1 and immediately pulsed with native soluble IMDQ, PASP, PEAD and PASP-IMDQ, as well as coacervated PEAD/PASP and PEAD/PASP-IMDQ at desired concentrations in six-fold. After $24 \mathrm{~h}$ incubation, $40 \mu \mathrm{L}$ of the MTT reagent $(1 \mathrm{mg} / \mathrm{mL})$ was added to the samples. The formed formazan crystals after an incubation period of 2-3h were dissolved in $100 \mu \mathrm{L}$ of a $10 \% \mathrm{~m} / \mathrm{v} \mathrm{SDS} / 0.01 \mathrm{M} \mathrm{HCl}$ solution overnight protected from light. The absorbance was measured by a microplate reader at $570 \mathrm{~nm}$. As a negative and positive control, PBS and DMSO were added to the wells, respectively.

In vivo innate immune activation. Heterozygous IFN-reporter mice (BALB/CIfnb1tm1.2Lien) in the range of 7-9 weeks were housed in individual ventilated cages and given ad libitum access to food and water. The mice were subjected to treatment with intradermal injection in the flank with a dose of $10 \mu \mathrm{g}$ (in $20 \mu \mathrm{L}$ of PBS) of PASP, PASP$I M D Q$, or IMDQ $(n=3)$. For in vivo imaging, mice were injected subcutaneously with 200 $\mu \mathrm{l}$ D-luciferin (15 mg/ml, Gold Biotechnology, USA) at the given time points (4, 24, and $48 \mathrm{~h}$ post injection), and in vivo luminescence imaging was recorded 12 min later using the IVIS Lumina II imaging system (PerkinEImer, USA). Local (flank) and full-body luminescence were quantified using the Living Image 4.4 software (Caliper life sciences, Hopkinton, MA). 
In vivo therapeutic anti-tumor response. CT-26 bearing female BALB/c mice were used to investigate the in vivo therapeutic anti-tumor response. In total, $5 \times 10^{5}$ CT- 26 cells suspended in $100 \mu \mathrm{l}$ of demi water were injected s.c. into the left flank of each $\mathrm{BALB} / \mathrm{c}$ mice, respectively. After inoculation of the tumor cells, the solid tumors were allowed to grow $\geq 100 \mathrm{~mm}^{3}$, then mice were divided into four groups (six mice per group): (1) demi water (Control); (2) IMDQ $(0.2 \mathrm{mg} / \mathrm{mL}) ;$ (3) PASP-IMDQ $(2 \mathrm{mg} / \mathrm{mL})$; (4) PEAD/PASP-IMDQ suspension.

The mice in each group were treated with the above formulations by intratumoral injection every 3 days. After the first administration, the tumors diameters were measured with calipers and the body weights of the mice were measured using an electronic balance every 3 days. The tumor volume was calculated using the following Eq.:

$$
V=\left(a^{*} b^{2}\right) / 2
$$

where $a$ and $b$ represent the length and width of the tumor, respectively. The mice were humanely euthanized when the volume of the tumor reached $10000 \mathrm{~mm}^{3}$.

\section{ACKNOWLEDGMENT}

H.L. acknowledges the Chinese "post-doctoral international exchange program" for a post- doctoral scholarship. L.N. kindly acknowledges financial support by the German Research Foundation (DFG) through the Emmy-Noether program and the SFB 1066 Project B04. Zifu Zhong acknowledges financial support from China Scholarship Council (CSC) (201607650018). S.D. acknowledges funding from NIAID Contract HHSN272201400056C (Adjuvant Discovery). Y.W. acknowledges financial support by Cornell Startup Grant. BGDG acknowledges Kom op Tegen Kanker for funding. Z.Z. acknowledges the Qilu Young Scholar program for funding. We thank Translational Medicine Core Facility of Advanced Medical Research Institute, Shandong University, for consultation and instrument availability that supported this work. 


\section{REFERENCES}

1. Adams, J. L.; Smothers, J.; Srinivasan, R.; Hoos, A., Big opportunities for small molecules in immuno-oncology. Nat Rev Drug Discov 2015, 14 (9), 603-622.

2. Suntharalingam, G.; Perry, M. R.; Ward, S.; Brett, S. J.; Castello-Cortes, A.; Brunner, M. D.; Panoskaltsis, N., Cytokine storm in a phase 1 trial of the anti-CD28 monoclonal antibody TGN1412. New Engl J Med 2006, 355 (10), 1018-1028.

3. Bjornmalm, M.; Thurecht, K. J.; Michael, M.; Scott, A. M.; Caruso, F., Bridging BioNano Science and Cancer Nanomedicine. Acs Nano 2017, 11 (10), 9594-9613.

4. Nicolas, J., Drug-Initiated Synthesis of Polymer Prodrugs: Combining Simplicity and Efficacy in Drug Delivery. Chem Mater 2016, 28 (6), 1591-1606.

5. Kawai, T.; Akira, S., The role of pattern-recognition receptors in innate immunity: update on Toll-like receptors. Nat Immunol 2010, 11 (5), 373-384.

6. Aderem, A.; Ulevitch, R. J., Toll-like receptors in the induction of the innate immune response. Nature 2000, 406 (6797), 782-787.

7. Liu, H. P.; Moynihan, K. D.; Zheng, Y. R.; Szeto, G. L.; Li, A. V.; Huang, B.; Van Egeren, D. S.; Park, C.; Irvine, D. J., Structure-based programming of lymph-node targeting in molecular vaccines. Nature 2014, 507 (7493), 519-522.

8. Brightbill, H. D.; Libraty, D. H.; Krutzik, S. R.; Yang, R. B.; Belisle, J. T.; Bleharski, J. R.; Maitland, M.; Norgard, M. V.; Plevy, S. E.; Smale, S. T.; Brennan, P. J.; Bloom, B. R.; Godowski, P. J.; Modlin, R. L., Host defense mechanisms triggered by microbial lipoproteins through toll-like receptors. Science 1999, 285 (5428), $732-$ 736.

9. Rodell, C. B.; Arlauckas, S. P.; Cuccarese, M. F.; Garris, C. S.; Ahmed, R. L. M. S.; Kohler, R. H.; Pittet, M. J.; Weissleder, R., TLR7/8-agonist-loaded nanoparticles promote the polarization of tumour-associated macrophages to enhance cancer immunotherapy. Nat Biomed Eng 2018, 2 (8), 578-588.

10. Albin, T. J.; Tom, J. K.; Manna, S.; Gilkes, A. P.; Stetkevich, S. A.; Katz, B. B.; Supnet, M.; Felgner, J.; Jain, A.; Nakajima, R.; Jasinskas, A.; Zlotnik, A.; Pearlman, E.; Davies, D. H.; Felgner, P. L.; Burkhardt, A. M.; Esser-Kahn, A. P., Linked Toll-Like Receptor Triagonists Stimulate Distinct, Combination-Dependent Innate Immune Responses. Acs Central Sci 2019, 5 (7), 1137-1145. 
11. Diebold, S. S., Recognition of viral single-stranded RNA by Toll-like receptors. Adv Drug Deliver Rev 2008, 60 (7), 813-823.

12. Kastenmuller, K.; Wille-Reece, U.; Lindsay, R. W. B.; Trager, L. R.; Darrah, P. A.; Flynn, B. J.; Becker, M. R.; Udey, M. C.; Clausen, B. E.; Igyarto, B. Z.; Kaplan, D. H.; Kastenmuller, W.; Germain, R. N.; Seder, R. A., Protective T cell immunity in mice following protein-TLR7/8 agonist-conjugate immunization requires aggregation, type I IFN, and multiple DC subsets. J Clin Invest 2011, 121 (5), 17821796.

13. Nuhn, L.; Vanparijs, N.; De Beuckelaer, A.; Lybaert, L.; Verstraete, G.; Deswarte, K.; Lienenklaus, S.; Shukla, N. M.; Salyer, A. C. D.; Lambrecht, B. N.; Grooten, J.; David, S. A.; De Koker, S.; De Geest, B. G., pH-degradable imidazoquinolineligated nanogels for lymph node-focused immune activation. P Natl Acad Sci USA 2016, 113 (29), 8098-8103.

14. Van Herck, S.; Deswarte, K.; Nuhn, L.; Zhong, Z.; Catani, J. P. P.; Li, Y.; Sanders, N. N.; Lienenklaus, S.; De Koker, S.; Lambrecht, B. N.; David, S. A.; De Geest, B. G., Lymph-Node-Targeted Immune Activation by Engineered Block Copolymer Amphiphiles-TLR7/8 Agonist Conjugates. J Am Chem Soc 2018, 140 (43), 1430014307.

15. Lynn, G. M.; Laga, R.; Darrah, P. A.; Ishizuka, A. S.; Balaci, A. J.; Dulcey, A. E.; Pechar, M.; Pola, R.; Gerner, M. Y.; Yamamoto, A.; Buechler, C. R.; Quinn, K. M.; Smelkinson, M. G.; Vanek, O.; Cawood, R.; Hills, T.; Vasalatiy, O.; Kastenmuller, K.; Francica, J. R.; Stutts, L.; Tom, J. K.; Ryu, K. A.; Esser-Kahn, A. P.; Etrych, T.; Fisher, K. D.; Seymour, L. W.; Seder, R. A., In vivo characterization of the physicochemical properties of polymer-linked TLR agonists that enhance vaccine immunogenicity. Nat Biotechnol 2015, 33 (11), 1201-1210.

16. Singh, M.; Khong, H.; Dai, Z. M.; Huang, X. F.; Wargo, J. A.; Cooper, Z. A.; Vasilakos, J. P.; Hwu, P.; Overwijk, W. W., Effective Innate and Adaptive Antimelanoma Immunity through Localized TLR7/8 Activation. J Immunol 2014, 193 (9), 4722-4731. 
17. Ignacio, B. J.; Albin, T. J.; Esser-Kahn, A. P.; Verdoes, M., Toll-like Receptor Agonist Conjugation: A Chemical Perspective. Bioconjugate Chem 2018, 29 (3), 587-603.

18. Lee, H. Y.; Jee, H. W.; Seo, S. M.; Kwak, B. K.; Khang, G.; Cho, S. H., Diethylenetriaminepentaacetic acid-gadolinium (DTPA-Gd)-conjugated polysuccinimide derivatives as magnetic resonance imaging contrast agents. Bioconjugate Chem 2006, 17 (3), 700-706.

19. Tomida, M.; Nakato, T.; Matsunami, S.; Kakuchi, T., Convenient synthesis of high molecular weight poly(succinimide) by acid-catalysed polycondensation of Laspartic acid. Polymer 1997, 38 (18), 4733-4736.

20. Lybaert, L.; Ryu, K. A.; De Rycke, R.; Chon, A. C.; De Wever, O.; Vermaelen, K. Y.; Esser-Kahn, A.; De Geest, B. G., Polyelectrolyte-Enrobed Cancer Cells in View of Personalized Immune-Therapy. Adv Sci 2017, 4 (6).

21. Chu, H. H.; Johnson, N. R.; Mason, N. S.; Wang, Y. D., A [polycation:heparin] complex releases growth factors with enhanced bioactivity. J Control Release 2011, 150 (2), 157-163.

22. Johnson, N. R.; Wang, Y. D., Controlled delivery of heparin-binding EGF-like growth factor yields fast and comprehensive wound healing. J Control Release 2013, 166 (2), 124-129.

23. Nuhn, L.; De Koker, S.; Van Lint, S.; Zhong, Z. F.; Catani, J. P.; Combes, F.; Deswarte, K.; Li, Y. P.; Lambrecht, B. N.; Lienenklaus, S.; Sanders, N. N.; David, S. A.; Tavernier, J.; De Geest, B. G., Nanoparticle-Conjugate TLR7/8 Agonist Localized Immunotherapy Provokes Safe Antitumoral Responses. Adv Mater 2018, $30(45)$.

24. Mullins, S. R.; Vogel, K.; Vasilakos, J.; Grigsby, I.; Dovedi, S.; Patricia, R.; Cooper, Z.; Herbst, R.; Kumar, R.; Tomai, M.; Wilkinson, R. W., Intratumoral immunotherapy with TLR7/8 agonist MEDI9197 modulates the tumor microenvironment and holds potential for combination with immune checkpoint inhibitors. Cancer Res 2017, 77. 
25. Shukla, N. M.; Malladi, S. S.; Mutz, C. A.; Balakrishna, R.; David, S. A., StructureActivity Relationships in Human Toll-Like Receptor 7-Active Imidazoquinoline Analogues. J Med Chem 2010, 53 (11), 4450-4465.

26. Ding, X. C.; Miller, P. G.; Hwang, M. P.; Fu, J. Y.; Wang, Y. D., Scale-up synthesis of a polymer designed for protein therapy. Eur Polym J 2019, 117, 353-362. 